\title{
V-shaped switching ferroelectric liquid crystal structure stabilized by dielectric surface layers
}

\author{
A. Hammarquist, ${ }^{1}$ K. D’Havé, ${ }^{1}$ M. Matuszczyk, ${ }^{2}$ N. A. Clark, ${ }^{3}$ J. E. Maclennan, ${ }^{3}$ and P. Rudquist ${ }^{1}$ \\ ${ }^{1}$ Photonics Laboratory, Department of Microtechnology and Nanoscience, \\ Chalmers University of Technology, S-412 96 Göteborg, Sweden \\ ${ }^{2}$ MC2 Process Laboratory, Department of Microtechnology and Nanoscience, \\ Chalmers University of Technology, S-412 96 Göteborg, Sweden \\ ${ }^{3}$ Liquid Crystal Materials Research Center, Physics Department, University of Colorado, Boulder, Colorado 80309, USA
}

(Received 30 November 2007; published 27 March 2008)

\begin{abstract}
The "V-shaped switching" mode in high polarization ferroelectric liquid crystals was studied with the aim of stabilizing the monostable bookshelf structure with the spontaneous polarization parallel to the glass plates. The director field in such cells was confirmed to be sensitive to both the liquid crystal properties and the cell parameters. In cells with only polyimide alignment layers, hysteresis free switching was never obtained, with bistable and asymmetric monostable structures compromising the zero-field dark state and preventing an ideal, hysteresis-free analog response. By incorporating a $\mathrm{SiO}_{2}$ layer between the ITO electrode and the polyimide, the undesired states were suppressed and essentially hysteresis-free switching was obtained for driving frequencies in the range $0.2-200 \mathrm{~Hz}$. Cells rubbed only on one side give more uniform alignment than cells rubbed on both sides but their inherent asymmetry shifts the long-term dark state away from $0 \mathrm{~V}$ and causes the response to gray level voltage modulation to be slightly asymmetric. The formation of different types of states as a function of the values of the surface parameters, and the observed stabilization of the V-shaped switching structure by the dielectric surface layers, are in good agreement with an earlier analysis by Copic et al. [Phys. Rev. E 65, 021701 (2002)].
\end{abstract}

DOI: 10.1103/PhysRevE.77.031707

PACS number(s): $61.30 . \mathrm{Hn}, 42.79 . \mathrm{Kr}$

\section{INTRODUCTION}

The analog electrooptic mode in certain chiral smectic liquid crystals with high spontaneous polarization, dubbed V-shaped switching after their transmission-voltage characteristics, gives thresholdless, high contrast response and seems ideal for active matrix displays [1,2]. This switching was first proposed to be the response of a new type of tilted chiral phase in which each smectic layer is similar to the smectic $C^{*}$ but where there is no correlation in tilt directions between the layers $[1,3]$. Such a smectic- $C_{R}^{*}(R$ for random) phase would be macroscopically uniaxial with its optic axis along the smectic layer normal, and have the same symmetry as the smectic- $A^{*}$ phase. This hypothetical variant of a "tilted" smectic $A$ was originally suggested by de Vries in the 1970 s as an elegant way of interpreting peculiar x-ray diffraction data for certain (nonchiral) materials not showing any layer shrinkage when cooling from the (nominally) orthogonal smectic- $A$ to the tilted smectic- $C$ phase [4]. In chiral smectic- $A$ materials of this kind, an electric field applied perpendicular to the smectic layer normal $\mathbf{z}$ would couple to the electric polarization of each layer and bias the director towards one side of the smectic cone, ideally without any increase in local tilt of the molecules [1,5-7]. This azimuthal biasing transforms the uniaxial medium to biaxial and results in an analog rotation of the apparent optic axis about the field direction. In "bookshelf" cells (with smectic layers aligned perpendicular to the cell substrates) this would give analog transmission-voltage curves [1].

In 1998 we demonstrated that the V-shaped switching effect did not occur in a random tilted smectic phase as first reported $[1,2]$ but rather in the conventional smectic- $C^{*}$ phase [8]. The electrooptic effect is caused by the usual field- induced rotation of the director on the surface of the smectic cone, where all positions on the cone are accessible. The key to obtaining thresholdless, monostable switching is the unusual zero field state of the surface-stabilized smectic- $C^{*}$ cell (i.e., with the helix suppressed by the surface action), in which the director is at the top (or the bottom) of the smectic cone, as shown in Fig. 1(a), rather than at the side as in conventional surface-stabilized ferroelectric liquid crystal (FLC) cells [9]. The projection of the director onto the cell plane in the absence of applied electric fields is thus along the smectic layer normal. This was demonstrated experimentally by observing the asymmetric change in effective birefringence when tilting the cell between crossed polarizers [10]. That $\mathrm{V}$ switching is a result of collective director reorientation was later independently confirmed by the Tokyo group [11].

The tilted director structure proposed for $\mathrm{V}$ switching cells depicted in Fig. 1(a) gives extinction when the cell is
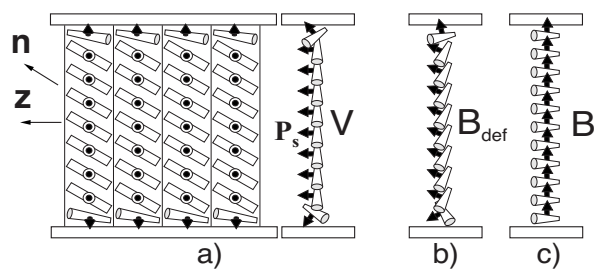

FIG. 1. Director structures in surface-stabilized SmC* cells. (a) V-shaped switching bookshelf structure viewed along (left) and perpendicular to (right) the smectic layers. In the absence of applied electric field, the director in the bulk of the cell is tilted uniformly down from the layer normal. (b) $B_{\text {def }}$ (splayed) and (c) $B$ (uniform) structures. The local direction of $\mathbf{P}_{\mathrm{s}}$ is indicated with black arrows. 
placed between crossed polarizers with the layer normal $\mathbf{z}$ along one of the polarizers. It is very different from bistable, surface-stabilized FLCs [9], where the director (ideally) adopts one of two degenerate states with the director parallel to the cell boundaries and $\mathbf{P}_{\mathrm{s}}$ pointing UP or DOWN, illustrated in Fig. 1(c). Indeed, the V-shaped switching structure is closer to the familiar or well-known monostable "twisted smectic- $C^{*}$ " or "SPLAYED" state $[12,13]$, where $\mathbf{P}_{\mathrm{s}}$ points into (or out of) the two surfaces, rotating smoothly through $180^{\circ}$ going from one surface to the other, with $\mathbf{P}_{\mathrm{s}}$ in the center of the cell being parallel to the glass. This smoothly twisted smectic- $C^{*}$ structure never produces a dark state between crossed polarizers but rather a characteristic bluish state. In materials with sufficiently high polarization density $\mathbf{P}_{s}$, however, the polarization charge self-interaction becomes important [10]. In this case any divergence in $\mathbf{P}_{\mathrm{s}}$ is expelled from the bulk, producing a homogeneous director field in the interior of the cell and two deformed surface regions ("polarization stabilized kinks") with thickness $\xi_{P}$ $=\left(\varepsilon_{\mathrm{LC}} K / P^{2}\right)^{1 / 2}$, where $\varepsilon_{\mathrm{LC}}$ is the dielectric constant [14] and $K$ the effective elastic constant $[15,10]$ of the liquid crystal. This state [shown in Fig. 1(a)] gives (1) extinction when the smectic layer normal is along one of the polarizers at zero field and (2) a thresholdless electrooptic response because of the nonzero torque $\mathbf{P} \times \mathbf{E}$ on $\mathbf{n}$ even in very small applied electric fields.

In order to obtain $\mathrm{V}$-shaped switching there must be a restoring torque for any deviation from the ground state. In the polarization-stabilized twisted structure this torque is provided by the polar surface anchoring [16]. It is thus evident that the $\mathrm{V}$-shaped switching phenomenon is not a property of all high polarization FLC materials but a result of the combination of liquid crystal properties and suitable liquid crystal-surface interactions.

Clark et al. have analyzed the surface energies required for the polarization-stabilized twisted structure to appear [17]. They demonstrated that in the absence of ions, which would otherwise partly screen the local polarization, the surfaces are typically too weak to hold the polar surface orientation in the polarization-splayed state for $P_{\mathrm{s}} \gtrsim 100 \mathrm{nC} / \mathrm{cm}^{2}$. The smectic- $C^{*}$ liquid crystal instead forms a block uniformly oriented from surface to surface, with $\mathbf{P}_{\mathrm{s}}$ reorienting under an applied voltage to screen the electric field in the liquid crystal completely. In the high polarization limit and in the absence of ions, neither surface nor elastic energies enter the problem and the director response becomes completely controlled by electrostatics in this model. Without the polar anchoring, the mechanical restoring torque is lost. Clark et al. showed, however, that when there are insulating layers between the conducting electrode and the liquid crystal, there is a restoring torque due to the buildup of electrostatic energy in these layers as soon as $\mathbf{P}_{\mathrm{S}}$ has a component perpendicular to the surfaces. The applied electric field reorients the local $\mathbf{P}_{\mathrm{s}}$ until compensated by the field from the polarization charges induced at the surfaces and the new equilibrium orientation is characterized [17] by

$$
V=2 t P \cos \phi / \varepsilon_{i},
$$

where $\phi$ is the angle between the spontaneous polarization and the applied field, and $t$ and $\varepsilon_{i}$ are respectively the thick-

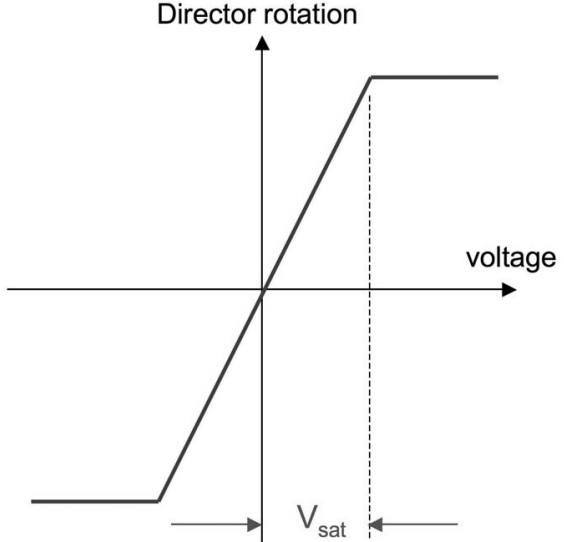

FIG. 2. Model director response in a smectic- $C^{*}$ cell with polarization stiffening and insulating surface layers (after [17]). The rotation of the director on the smectic cone (and hence the optical response) saturates at the voltage $V_{\text {sat }}$, for which the polarization is parallel to the electric field.

ness and dielectric constant of the insulating surface layers. For $|V|>V_{\text {sat }}=2 t P / \varepsilon_{i}$ the switching is saturated $(\phi=0$ or $\phi$ $=180^{\circ}$ ), and with increasing applied voltage, a voltage drop appears over the LC. Accordingly, the voltage $V_{\text {sat }}$ for complete switching (see Fig. 2) or the "width of the V," is proportional to the thickness $t$ and to the polarization $\mathbf{P}_{\mathrm{S}}$ behavior which has been verified experimentally [18]. The effect of dielectric surface layers on the V-shaped switching have also been investigated by Rudquist et al. [8,10,19], Takezoe et al. [20,21], Chandani et al. [22], Hammarquist [23], Blinov et al. [24], and recently by Vij et al. [25]. A modification of the twisted structure, involving a twist of the smectic layers close to the substrate surfaces, was proposed by Ikeda et al. [26].

Copic et al. performed a detailed analysis of the effects of anchoring conditions (polar and nonpolar) and the thickness of the alignment layers on different surface-stabilized states, for small and intermediate $P_{\mathrm{s}}$ [27]. It turns out that depending on the polar properties of the surfaces, and their thickness and dielectric permittivity, a plethora of bistable and monostable FLC states are possible, and moreover, different states can coexist in the same cell. Importantly, the model showed that for intermediate values of $P_{\mathrm{s}}$ and with thick insulating surface layers, the $\mathrm{V}$-shaped switching structure is likely to be a compromise between the polarizationstabilized twisted structure [10] and the homogeneous block structure [17] of Fig. 1(a). This could be described as a polarization-stabilized twisted smectic- $C^{*}$ structure with a high pretilt of $\mathbf{n}$ at the surfaces. It was evident from this study that a thorough understanding of how to control the surface parameters would be necessary to exploit $\mathrm{V}$-shaped switching.

O'Callaghan studied the switching dynamics of the $\mathrm{V}$-shaped switching mode away from the high polarization limit [28]. He suggested that the surprisingly slow switching speeds (and hysteresis effects) found in some analog switching FLC cells are a result of the vanishing electric torque on $\mathbf{P}_{\mathrm{s}}$ when the director approaches the two extreme positions 


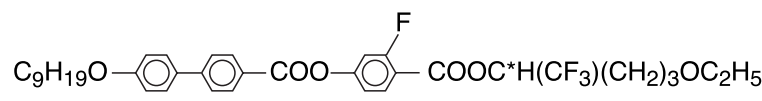

FIG. 3. Structure of the liquid crystal MC-452. The phase sequence is isotropic $-102{ }^{\circ} \mathrm{C}-\mathrm{Sm} A^{*}-100{ }^{\circ} \mathrm{C}-\mathrm{Sm} C^{*}-\left(\sim 35^{\circ} \mathrm{C}\right)-$ crystal.

on the smectic cone, i.e., close to the fully switched states. To overcome this problem O'Callaghan proposed charge controlled drive $[29,30]$, rather than voltage-controlled drive, to speed up the response and to suppress apparent hysteresis effects.

Many reports on $\mathrm{V}$-shaped switching in the literature are based on the response to triangular voltages at very low frequencies, often in the $\mathrm{mHz}$ regime. Analog, domain-free response at these frequencies may indeed prove that there is no threshold, and confirm, say, that the material is not antiferroelectric, but gives little information about the performance at typical addressing frequencies, for example in video frame rate applications. Moreover, at low frequencies, ions in the liquid crystal have time to drift and build up a counteracting electric field opposing the applied external field. The local field inside the liquid crystal may then lead the applied external field and the transmission versus (external) voltage curve can look $\mathrm{V}$-shaped, even if there is significant hysteresis of the switching relative to the internal field [31-33]. Blinov et al. have argued [33] that hysteresis-free V-shaped electrooptical switching is only an "apparent phenomenon" resulting from the capacitance of the alignment layer and the capacitance and resistance of the ferroelectric liquid crystal layer-the FLC itself always has a well-pronounced threshold and a normal hysteresis. This scenario is however not consistent with thresholdless, domain-free switching with a zero-field dark state "at the tip of the V" at very low frequencies (even down to dc). In this paper we present experimental results that confirm that for true $\mathrm{V}$-shaped switching, which gives a well-controlled gray scale as a function of the amplitude of the applied voltage and extinction at $V=0$, we must stabilize the structure of Fig. 1(a).

\section{EXPERIMENT}

We have studied the single component material MC-452 (Mitsubishi Chemical Gas Company, Japan), shown in Fig. 3. This material can be supercooled to below room temperature and at room temperature crystallization often occurs only after several days. The sample cells are of sandwich type, with ITO electrodes and rubbed polyimide as alignment layers. For cells with additional dielectric surface layers we evaporated $\mathrm{SiO}_{2}$ onto the ITO before spin-coating with polyimide. The polyimide layer was rubbed using a commercial rubbing machine (LC Tec Automation). Surface processing and cell assembly were carried out in-house on $3 \times 3$ in. $^{2}$ ITO glass substrates using PI2610 (Pyralin, Dupont) to make alignment layers about $40 \mathrm{~nm}$ thick (as determined from Alphastep measurements). The substrates were then glued together using a UV-hardening glue (NOA68) with the cell gap defined by spacer balls mixed into the glue. The final cell thickness was estimated from the interference spectrum obtained using a spectrophotometer. From each assembly we cut out 25 identical sample cells, allowing for reliable reproduction of the experiments. The cells were capillary-filled with the LC material in the isotropic phase. The phase sequence of MC-452 was determined by polarizing microscopy using a hot stage (Linkam), and the apparent tilt angle $\theta$ was measured from the electrooptic switching between crossed polarizers. The spontaneous polarization $\mathbf{P}_{\mathrm{s}}$ was estimated from the area of the polarization peak in the current signal for saturated switching with triangular voltages.

The director tilt jumps from $0^{\circ}$ to about $27^{\circ}$ at the (first order) $\mathrm{Sm} A^{*}$ to $\mathrm{Sm}^{*}$ transition and saturates at about $39^{\circ}$ at room temperature. The polarization increases approximately linearly on cooling, from about $150 \mathrm{nC} / \mathrm{cm}^{2}$ at the $A^{*}$ to $C^{*}$ transition to about $400 \mathrm{nC} / \mathrm{cm}^{2}$ at room temperature. The optical response time (for a $10 \mathrm{~V}$ peak-to-peak square wave) decreases from about $2 \mathrm{~ms}$ at room temperature to about $50 \mu \mathrm{s}$ at the transition to the smectic- $A^{*}$ phase. Measurements of tilt, polarization, and response time are reported in Ref. [23].

\section{RESULTS AND DISCUSSION}

In our cells, MC-452 exhibits a significant surface electroclinic effect [34-36], resulting in a rotation $\alpha \approx 10^{\circ}$ of the bulk smectic layer normal $\mathbf{z}$ from the rubbing direction. In cells with the two surfaces rubbed in parallel, a random twodomain structure appeared, with the layer orientation of the two sets of domains controlled either by the top or by the bottom surface. In order to avoid this indeterminacy in layer orientation, we prepared cells with the two surfaces buffed at an angle $2 \alpha$ with respect to each other, a technique known as cross-rubbing [26,37-40]. In this way, we did obtain a homogeneous bookshelf structure when cooling from isotropic to $\operatorname{Sm} A^{*}$, but this structure still tended to break up into a two-domain structure in the $\mathrm{SmC} C^{*}$ phase when an electric field was applied. This could be related to the process of straightening up of the original vertical chevrons, during which the layers often instead form "horizontal chevrons" [41]. Better alignment stability was found in cells with only one side rubbed, in which there is no conflict in layer orientation from the SEC effect but where the asymmetric boundary conditions have other consequences for the electrooptics, as will be discussed below.

Typical textures and electrooptic responses obtained in a $1.55 \mu \mathrm{m}$ thick, single-side rubbed cell without $\mathrm{SiO}_{2}$ layers are shown in Fig. 4. We essentially see two types of regions: type 1 with an average optic axis more or less along $\mathbf{z}$ at zero field (dark), and type 2 with the apparent optic axis rotated to $\theta \approx 20^{\circ}$ from $\mathbf{z}$ (bright). By passing a laser ( $\mathrm{HeNe}, \lambda$ $=540 \mathrm{~nm}$ ) through the polarizing microscope, we could measure separately the electrooptic response of the two regions to triangular ac voltages of $1-100 \mathrm{~Hz}$. The regions exhibit quite different electrooptic responses. Type 1 regions show a "W-shaped" transmission-voltage response, with "normal apparent hysteresis," i.e., the electrooptic response lags the external field and the hysteresis increases with higher frequency. This response could incorrectly be interpreted to be 

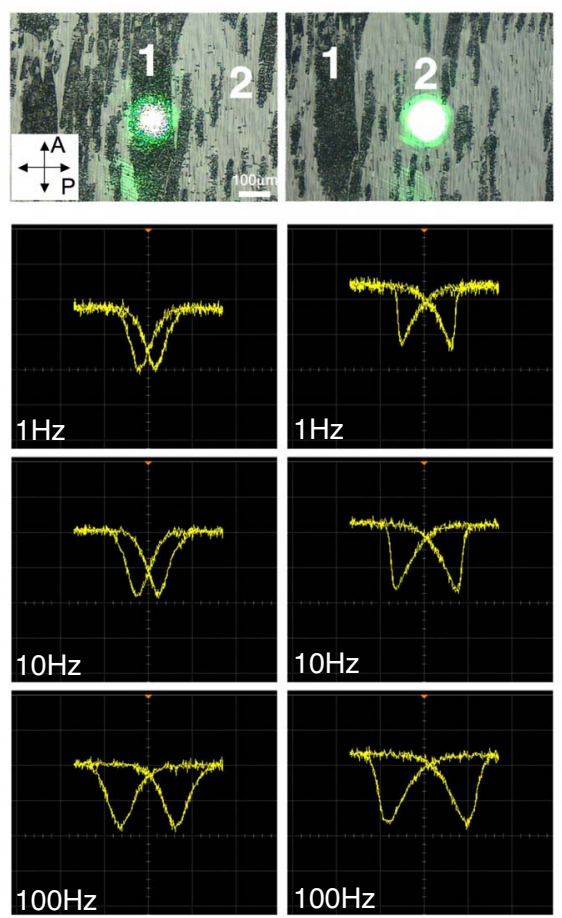

Type 1

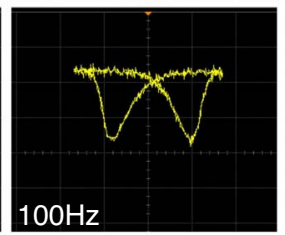

Type 2

FIG. 4. (Color online) Top: Two-domain texture in $1.5 \mu \mathrm{m}$ thick cell of MC-452 without $\mathrm{SiO}_{2}$ layers at zero field. Region 1 is dark (average optic axis along the polarizer) whereas region 2 is bright (optic axis tilted away from the smectic layer normal and the polarizer). Bottom: Transmission vs voltage $(T-V)$ curves for triangular ac voltages at $1 \mathrm{~Hz}, 10 \mathrm{~Hz}$, and $100 \mathrm{~Hz}\left(35 V_{p p}\right)$, for region 1 (left) and region 2 (right). The $T$ - $V$ curves were recorded at room temperature using a green laser beam passing through a polarizing microscope, the usual microscope illumination being blocked while the measurements were carried out.

V-shaped switching with hysteresis. However, the grainy appearance of the type 1 regions suggests that the apparently thresholdless response is rather an averaged result of many bistable microdomains switching at slightly different voltages and times. We propose that these domains could be examples of the different bistable structures $B\left(\mathbf{P}_{\mathrm{s}}\right.$ essentially perpendicular to the cell plane) and $B_{\text {def }}\left(\mathbf{P}_{\mathrm{s}}\right.$ at an angle with respect to the cell plane) discussed in [27] and illustrated in Figs. 1(b) and 1(c).

Type 2 regions show large hysteresis even at $1 \mathrm{~Hz}$. The apparent tilt of $\theta=20^{\circ}$ of the zero-field optic axis with respect to the layer normal is significantly less than half the cone angle $2 \psi_{0} \approx 78^{\circ}$ [23]. If we assume perfect bookshelf layer structure, this implies that $\mathbf{n}$ is not parallel to the surface and that $\mathbf{P}_{\mathrm{s}}$ makes an angle of about $30^{\circ}$ with respect to the cell plane. Importantly, the switching hysteresis of both 1 and 2 increase rapidly when the frequency is increased to tens of $\mathrm{Hz}$, as shown in Fig. 4. During slow switching, the motion of domain boundaries between polarization UP and DOWN domains was clearly visible.

Regions of type 1 and 2 formed immediately on cooling through the smectic- $A^{*}$ to smectic- $C^{*}$ transition and were unchanged on further cooling to room-temperature. Thus, the surface states adopted at the transition, which are responsible for the type of domains formed, are maintained. There was, however, a tendency for the type 1 domain to grow at the expense of type 2 on heating toward the smectic $A^{*}$ while fully switching the cell, indicating that it is possible to break the surface anchoring with applied electric fields.

Copic et al. treated the case of perfectly symmetric cells [27], i.e., with identically treated surfaces. In symmetric cells, all structures in Fig. 1 have a "twin-state" with opposite polarization so that these structures are bistable. The perfect $\mathrm{V}$ domain of Fig. 1(a) also has a twin state, with $\mathbf{P}_{\mathrm{s}}$ pointing to the left instead of to the right. If we were to apply fields in the plane of the cell, instead of perpendicular to the glass, we would be able to switch between the stable LEFTRIGHT polarization states. The $\mathrm{V}$ domain is therefore truly ferroelectric but when used for $\mathrm{V}$-shaped switching it is addressed in a thresholdless dielectric mode. Our cells are always slightly asymmetric, as a result of the single-side rubbing, so there are no twin states and we cannot directly compare our observations to the structures discussed in [27]. For example, what would have been a perfect $\mathrm{V}$ domain [Fig. $1(\mathrm{a})]$ in a symmetric cell will, in a nonsymmetric cell (with the slightest difference in pretilt on the two surfaces), tilt $\mathbf{P}_{\mathrm{S}}$ in the interior of the cell away from being parallel to the plates. The resulting structure would then be reminiscent of a (monostable) $B_{\text {def }}$ domain [Fig. 1(b)]. Furthermore, a (bistable) $B$ domain could in a nonsymmetric cell resemble a (monostable) $B_{\text {def }}$ domain but with $\mathbf{P}_{\mathrm{s}}$ nearly perpendicular to the glass plates. The large hysteresis, and the significant optic axis tilt at zero field, lead us to conclude that type 2 regions do not correspond to $\mathrm{V}$ domains in symmetric cells, but rather to the monostable analogue of $B$ or $B_{\text {def }}$. Importantly, we did not obtain the desired $\mathrm{V}$ domains in these cells, and hence no V-shaped switching was observed. Copic's analysis [27] suggested that one can promote the $\mathrm{V}$ domain structure by (i) increasing the strength of the polar surface anchoring and/or (ii) increasing the thickness $t$ of the insulating alignment layers.

Polar anchoring is used here as being proportional to $\mathbf{P} \cdot \hat{s}$, where $\hat{s}$ is the cell surface normal [12]. Very little is understood about how to control the strength of polar anchoring by tuning the surface parameters. We do know that strong polar anchoring tends to favor a "twisted" smectic- $C^{*}$ structure in bookshelf geometry, and that the polarization-stabilized twisted structure is one way of obtaining a $\mathrm{V}$ domain. We should then be able to increase the "apparent polar anchoring strength" by matching the rubbing directions, which essentially control the orientation of $\mathbf{n}$, with the different states (molecular tilt directions) promoted by the polar anchoring at the two surfaces. This means that the cross-rubbing directions on the two surfaces should make an angle $2 \theta \approx 78^{\circ}$ (the full smectic cone angle). However, with such a large crossrubbing angle it turned out to be very difficult to obtain a homogeneous bookshelf structure at the isotropic to $\operatorname{Sm} A^{*}$ transition. The optimization of cross-rubbing angles to compensate for the surface-electroclinic effect and to increase the apparent polar anchoring strength thus remains an interesting subject of further study.

Since we cannot yet control the (real or apparent) polar anchoring strength, we focused on varying the thickness of 


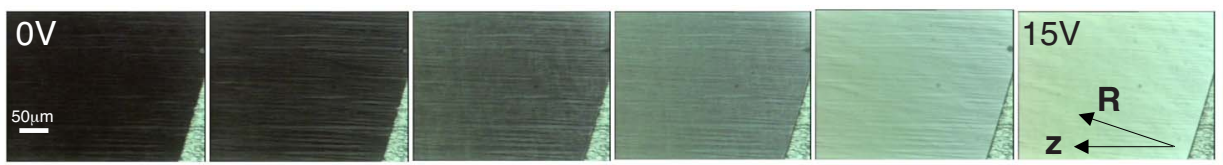

FIG. 5. (Color online) Static gray scale variation with increasing dc voltage in a $1.5 \mu \mathrm{m}$ thick cell of $\mathrm{MC}-452$ with $1000 \AA \mathrm{SiO}{ }_{2}$ layers. The (single-side) rubbing direction $\mathbf{R}$ is perpendicular to the ITO electrode edge visible in the lower right corner. The smectic layer normal $\mathbf{z}$ is horizontal, revealing a significant surface electroclinic effect. The applied voltage is varied from 0 to $15 \mathrm{~V}$. Outside the electrode area, where the vertical chevrons have not been straightened out, we have (permanent) bistable domains with $P_{\mathrm{UP}}$ and $P_{\mathrm{DOwN}}$, respectively.

the alignment layers. Cells including an additional $1000 \AA$ $\mathrm{SiO}_{2}$ layer were made according to the scheme described above and rubbed on only one surface. These cells exhibited a texture and electrooptic response very different from the cells without $\mathrm{SiO}_{2}$ layers, as shown in Figs. 5 and 6. After applying a large ac field in order to straighten out the vertical chevrons, the electrode area adopted an essentially homogeneous layer-director structure, with good extinction with the smectic layer normal parallel to the polarizer at $E=0$, as shown in Fig. 5, and perfect analog (grayscale) response. The electrooptic response under triangular ac voltages was practically hysteresis-free from $\sim 0.2 \mathrm{~Hz}$ up to about $200 \mathrm{~Hz}$ [Fig. 6(a)]. Outside this frequency range the response was characterized by larger hysteresis. Only "normal" hysteresis was observed (i.e., there was no inverse hysteresis at low frequencies) and so there was no inversion frequency. When the ac field was switched off, we always initially obtained excellent extinction with the smectic layer normal along the polarizer. This shows that immediately after switching off the field the director in the bulk returns, as desired, to the bottom (or top) of the smectic cone, with $\mathbf{P}_{\text {s }}$ parallel to the cell plane, as illustrated in Fig. 1(a). This orientation was confirmed by tilting the cell back and forth [8]. This dark state relaxed slowly (in tens of seconds to minutes) to a slightly less dark state. Good extinction could be obtained again by rotating the cell slightly in the microscope, indicating that the director tends to relax away from the original position at the top
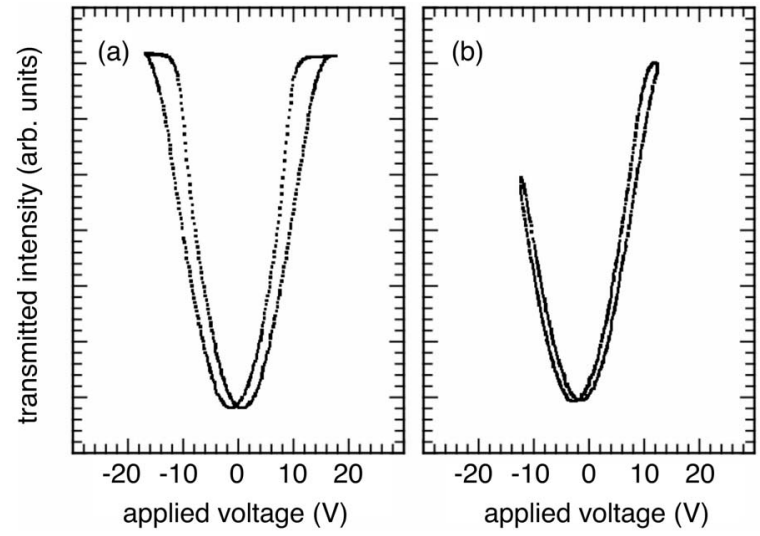

FIG. 6. Electrooptic response of the $\mathrm{SiO}_{2}$ cell in Fig. 5 to a $200 \mathrm{~Hz}$ triangular voltage. (a) $V_{p p}=35 \mathrm{~V}$ : There is slight hysteresis but the response curve is centered around $V=0$. (b) $V_{p p}=24 \mathrm{~V}$ : When the amplitude of the driving signal is smaller, the asymmetry of the single-side rubbed cell is manifest as a shift of the minimum away from $V=0$, and by different levels of transmission for negative and positive voltages. (or bottom) of the smectic cone. The original dark state with $\mathbf{z}$ parallel to one of the polarizers could be recovered by electrically addressing the cell and switching off the field. We attribute the slight relaxation to the fact that the singleside rubbed cell is inherently nonsymmetric as discussed before. This asymmetry also becomes apparent when switching the cell at lower amplitude [see Fig. 6(b)], where the electrooptic response is not saturated. The minimum in the transmission-voltage curve is shifted away from $V=0$ in this case and the magnitude of the response is different for positive and negative voltages. The reason the low-voltage curve does not overlap the high-voltage curve could be the following. For low-voltage (unsaturated) switching, the molecules at the surfaces do not switch and we have reorientation only of the bulk. The field for which the transmission is a minimum depends on the director configuration, which is itself controlled by the asymmetric pretilts at the cell surfaces, as in Fig. 1(b). For high-voltage (saturated) switching, we suggest that the surface regions also reorient, the FLC maintaining a homogeneous block structure everywhere. When the surface anchoring is overcome, the switching is essentially governed by electrostatics, as discussed in [17].

We have assumed here that any vertical chevron structures [42] have been fully straightened out with the applied electric fields. If, on the other hand, there were still a chevron (with small chevron angle, quasibookshelf structure), the bulk state with the director uniformly on the top or bottom of the smectic cone could be unstable. The presence of a chevron interface might therefore slightly shift the apparent equilibrium position of the optic axis in the cell. Furthermore, as we can see in Fig. 4 there are traces of zig-zag defects indicating that in some areas in cells without $\mathrm{SiO}_{2}$ layers, chevrons might still be present. It is not clear whether an internal chevron interface might contribute to the slight hysteresis observed in the $T-V$ curve of cells with $\mathrm{SiO}_{2}$ layers (shown in Fig. 6), although no zig-zag defects were visible in this cell after applying electric fields to straighten the chevrons. The effect of the chevron interface in stabilizing states with $\mathbf{P}_{\mathrm{s}}$ UP or DOWN is evident in Fig. 5, in the area outside the electrodes.

In $\mathrm{SiO}_{2}$ cells, we sometimes observe domains, as shown in Fig. 7. With normal microscope illumination, the domains could not be distinguished except by their visible boundaries. By using directional filtering [43] to obtain obliquely incident light we could identify these domains as regions in which the director is either at the bottom or the top of the smectic cone. These are therefore bistable ferroelectric domains (twin states) with lateral spontaneous polarization $\mathbf{P}_{\mathrm{s}}$ pointing in opposite directions (LEFT-RIGHT) in the plane 


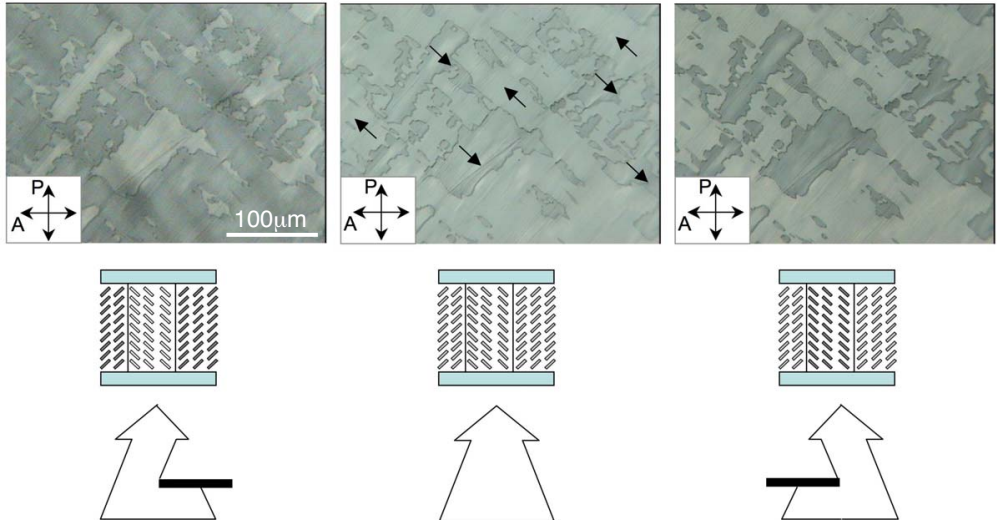

FIG. 7. (Color online) With the cell rotated $45^{\circ}$ with respect to the position giving $\mathrm{V}$-shaped response oblique illumination reveals domains with the director either at the top or at the bottom of the smectic cone. The domains through which the light travels "along" the director appear darker (smaller retardation) than the domains through which the light travels "perpendicular" to the director (larger retardation). The directional filtering was performed by blocking part of the microscope illumination with a screen. The spontaneous polarization is parallel to the glass plates and points in opposite directions (indicated with arrows) in adjacent domains. of the cell, as discussed above. At low driving voltages these domains switch in an analog dielectric mode due to the $\mathbf{P}$ $\times \mathbf{E}$ torque, with no domain wall motion. For saturated switching, the domains become indistinguishable when $\mathbf{P}_{\mathrm{s}}$ aligns parallel to the field everywhere, the destruction or formation of the domain walls probably contributing to the observed hysteresis. The domain walls also decrease the optical contrast. In order to obtain a monodomain in V-shaped switching FLC cells, it is important to make the LEFTRIGHT polarization states nondegenerate. Here control of pretilt angles and cross-rubbing angles, and polar vs nonpolar anchoring stand out as important issues.

The additional dielectric surface layers increase the required driving voltages for saturated switching as there is a voltage drop across them. In the linear region of the $T$ - $V$ response the voltage drop occurs essentially only over the surface layers. In the bulk of the cell, the polarization (ideally) screens the applied field, so that it vanishes inside the cell. This scenario was confirmed by measuring the width of the $\mathrm{V}$ as a function of the thickness of the dielectric surface layers. The results are in good agreement with theory [17] and similar investigations on other FLC systems $[18,22,44]$.

Figure 8(a) shows the electrooptic response of an $\mathrm{SiO}_{2}$ cell to arbitrary addressing waveforms. The perfect grayscale modulation obtained shows that all director states on the smectic cone are accessible and stable under dc fields. The switching or relaxation time between gray levels is about $1 \mathrm{~ms}$. A detailed examination of the stepwise switching waveform and the response [Fig. 8(b)] reveals that the response time is a function of the starting position on the smectic tilt cone: The switching seems slightly slower close to the saturated states, as suggested by O'Callaghan [28].

Finding a way of using symmetric cells in practical $\mathrm{V}$-shaped switching devices remains a challenge. Obtaining a perfect $\mathrm{V}$-domain with $\mathbf{P}_{\mathrm{s}}$ exactly parallel to the cell surfaces, i.e., with the director exactly at the bottom or top of the smectic cone, in asymmetric (single side-rubbed) cells is nontrivial. Moreover, in asymmetrically prepared cells the electrooptic response is asymmetric, at least for grayscale modulation as shown in Fig. 6(b), which may cause flicker in display devices. Further investigation will be necessary to determine how to achieve an ideal, symmetric response. The challenge would be to create symmetric surface conditions that compensate for the surface electroclinic effect, stabilize this alignment in the tilted smectic- $C^{*}$ phase, and promote the correct polar state at the surfaces required for monostable V-shaped switching. O'Callaghan has already demonstrated high-speed analog switching in V-FLC cells with response
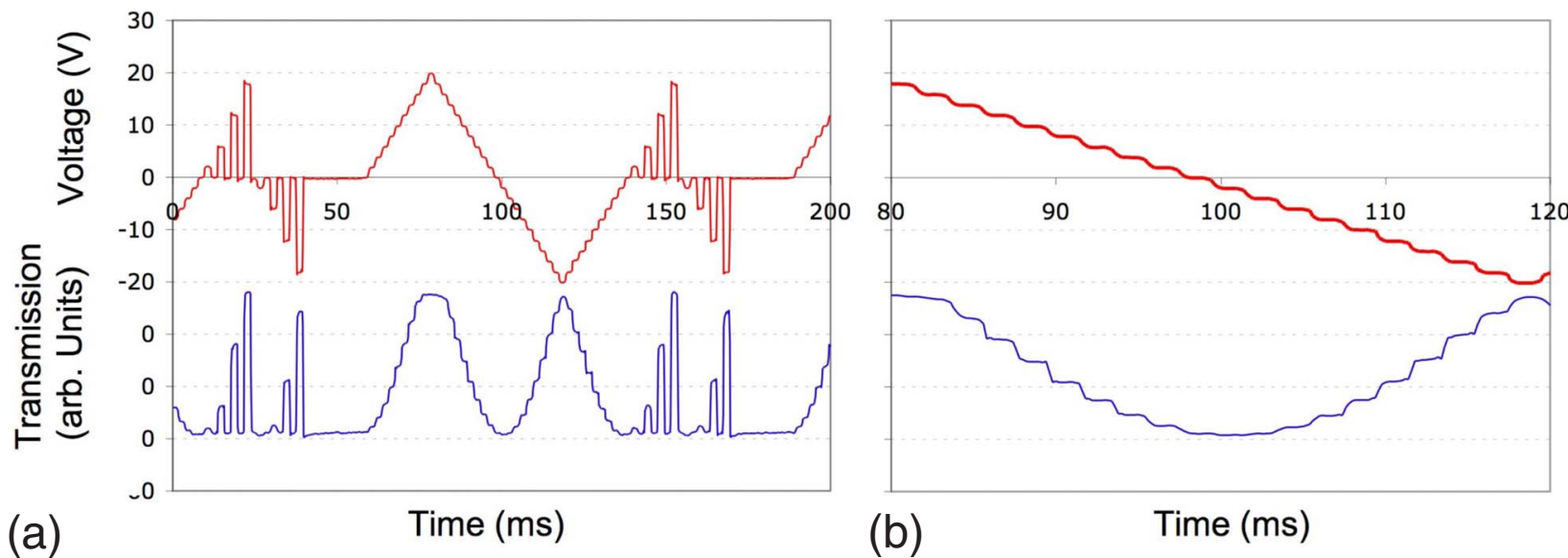

(b)

Time (ms)

FIG. 8. (Color online) (a) Electrooptic response (lower curve) of MC-452 in $1.5 \mu m$ cell with dielectric surface layers to arbitrary driving waveforms (upper curve) of maximum amplitude $20 \mathrm{~V}$. The response is slightly asymmetric, due to the single-side rubbing; cf. Fig. 6(b). (b) Detail of the optical response during the staircase part of the driving waveform. 
times in the sub-millisecond regime, utilizing chargecontrolled drive [29].

\section{CONCLUSIONS}

We have investigated experimentally polarizationstabilized V-shaped switching in ferroelectric liquid crystal cells with different dielectric layers. At zero field, the classical V-structure has the polarization essentially parallel to the cell surface, with the director either on the top $\left(\mathbf{P}_{\mathrm{s}}\right.$ pointing LEFT) or at the bottom $\left(\mathbf{P}_{\mathrm{s}}\right.$ pointing RIGHT) of the smectic$C$ tilt cone. Walls between such polarization domains cause light leakage and reduce the contrast in V-shaped switching devices. We have shown that additional insulating surface layers promote the structure that gives $\mathrm{V}$-shaped switching and suppress unwanted states with UP-DOWN polarization which show neither the static dark state nor a thresholdless electrooptic response. These results are in very good agree- ment with theoretical work by Copic et al. [27]. In cells prepared without additional surface layers, we did not obtain the desired zero-field dark state but two types of structures which we suggest could be examples of the $B$ and $B_{\text {def }}$ states described by Copic. These experiments confirm that $\mathrm{V}$-shaped switching in FLCs is not an intrinsic property of the liquid crystal material alone but a result of the judicious combination of liquid crystal material and the architecture of the cell.

\section{ACKNOWLEDGMENTS}

This work was supported by the Swedish Foundation for Strategic Research Grants No. 2002/0388 and No. 2005/ 0248, and by National Science Foundation Grant No. DMR0603223. The authors would also like to thank M. Johno at Mitsubishi Gas Chemical Company for providing the sample of MC-452 and M. Wessling for designing and assembling the laser measurement system.
[1] S. Inui, N. Iimura, T. Suzuki, T. Iwane, K. Miyachi, and A. Fukuda, J. Mater. Chem. 61, 177 (1996).

[2] T. Saishu, K. Takatoh, R. Iida, H. Nagata, and Y. Mori, SID Int. Symp. Digest Tech. Papers 27, 703 (1996).

[3] A. Fukuda, S. S. Seomun, T. Takanishi, Y. Takanishi, and K. Ishikawa, Mol. Cryst. Liq. Cryst. Sci. Technol., Sect. A 303, 379 (1997).

[4] A. De Vries, Mol. Cryst. Liq. Cryst. 41, 27 (1977).

[5] N. A. Clark, T. Bellini, R.-F. Shao, D. Coleman, S. Bardon, D. R. Link, J. E. Maclennan, X.-H. Chen, M. D. Wand, D. M. Walba, P. Rudquist, and S. T. Lagerwall, Appl. Phys. Lett. 80, 4097 (2002).

[6] Y. P. Panarin, V. Panov, O. E. Kalinovskaya, and J. K. Vij, J. Mater. Chem. 9, 2967 (1999).

[7] J. V. Selinger, P. J. Collings, and R. Shashidar, Phys. Rev. E 64, 061705 (2001).

[8] P. Rudquist, J. E. Maclennan, R. Shao, D. R. Link, D. M. Walba, and N. A. Clark, 17th International Liquid Crystal Conference 1998, Poster Presentation, P2-063 (unpublished); N. A. Clark, cited in A. Fukuda, Liq. Cryst. Today 8, 6 (1998).

[9] N. A. Clark and S. T. Lagerwall, Appl. Phys. Lett. 36, 899 (1980)

[10] P. Rudquist, J. P. F. Lagerwall, M. Buivydas, F. Gouda, S. T. Lagerwall, N. A. Clark, J. E. Maclennan, R. Shao, D. A. Coleman, S. Bardon, T. Bellini, D. R. Link, G. Natale, M. A. Glaser, D. M. Walba, M. D. Wand, and X.-H. Chen, J. Mater. Chem. 9, 1257 (1999).

[11] B. Park, S. S. Seomun, M. Nakata, M. Takahashi, Y. Takanishi, and H. Takezoe, Jpn. J. Appl. Phys., Part 1 38, 1474 (1999).

[12] M. Handschy, N. A. Clark, and S. T. Lagerwall, Phys. Rev. Lett. 51, 471 (1984).

[13] Strictly speaking, the SPLAYED state can also be bistable, provided the two states where the director rotates on the upper and lower part of the smectic cone going from one surface to the other are degenerate.

[14] $\varepsilon_{\mathrm{LC}}=\varepsilon_{\text {total }}-\varepsilon_{\mathrm{Ps}}$, the part of the dielectric constant which is not related to the reorientation of the spontaneous polarization $\mathbf{P}_{\mathrm{s}}$.

[15] Z. Zhuang, J. E. Maclennan, and N. A. Clark, Proc. SPIE 1080, 110 (1989).

[16] The torque arises from the elastic and electrostatic interactions that make it hard to compress the surface distortions and reorient the bulk but of course without surface anchoring this all would not matter much.

[17] N. A. Clark, D. A. Coleman, and J. E. Maclennan, Liq. Cryst. 20, 985 (2000).

[18] D. Coleman, D. Mueller, N. A. Clark, J. E. Maclennan, R.-F. Shao, S. Bardon, and D. M. Walba, Phys. Rev. Lett. 91, 175505 (2003).

[19] P. Rudquist, R. F. Shao, D. Coleman, S. Bardon, D. R. Link, T. Bellini, J. E. Maclennan, X. H. Chen, D. M. Walba, J. P. F. Lagerwall, M. Buivydas, F. Gouda, S. T. Lagerwall, and N. A. Clark, Society for Information Display 1999 International Symposium, Proceedings of the 1999 SID International Symposium, May 1999, San Jose, CA (SID, San Jose, 1999), pp. 409-413.

[20] Y. Takanishi, A. D. L. Chandani, Y. Cui, S. S. Seomun, K. Ishikawa, H. Takezoe, and A. Fukuda, Book of Abstracts, 17th International Liquid Crystal Conference, Strasbourg, 1998 (unpublished), pp. 1-57.

[21] H. Takezoe, A. D. L. Chandani, S. S. Seomun, B. Park, D. S. Hermann, Y. Takanishi, and K. Ishikawa, Surface Sensitive $V$-Shaped Switching in Antiferroelectric and Ferroelectric Liquid Crystals, Workshop Digest of the 18th International Display Research Conference, Asia Display '98, Seoul, Korea, 1998 (SID, Seoul, Korea, 1998), pp. 23-30.

[22] A. D. L. Chandani, Y. Cui, S. S. Seomun, Y. Takanishi, K. Ishikawa, and H. Takezoe, Liq. Cryst. 26, 167 (1999).

[23] A. Hammarquist, MSc thesis, Chalmers University of Technology, Gothenburg, Sweden (2004).

[24] L. M. Blinov, S. P. Palto, E. P. Pozhidaev, Y. P. Bobylev, V. M. Shoshin, A. L. Andreev, and F. V. Podgornov, Phys. Rev. E 71, 051715 (2005). 
[25] V. Manjuladevi, Yu. P. Panarin, and J. K. Vij, Appl. Phys. Lett. 91, 052911 (2007).

[26] S. Ikeda, T. Ogasawara, M. Nakata, Y. Takanishi, K. Ishikawa, and H. Takezoe, Phys. Rev. E 63, 061703 (2001).

[27] M. Copic, J. E. Maclennan, and N. A. Clark, Phys. Rev. E 65, 021708 (2002).

[28] M. J. O'Callaghan, Phys. Rev. E 67, 011710 (2003).

[29] M. J. O'Callaghan, Presentation at the 20th Ferroelectric Liquid Crystal Conference, Stare Jablonki, Poland, 2005 (unpublished).

[30] M. J. O'Callaghan, M. D. Wand, C. M. Walker, and M. Nakata, Appl. Phys. Lett. 85, 6344 (2004).

[31] P. Rudquist, D. Krüerke, S. T. Lagerwall, J. E. Maclennan, N. A. Clark, and D. M. Walba, Ferroelectrics 246, 927 (2000).

[32] Y. Panarin, V. Panov, O. E. Kalinovskaya, and J. K. Vij, Ferroelectrics 246, 35 (2000).

[33] L. M. Blinov, E. P. Pozhidaev, F. V. Podgorov, S. A. Pikin. S. P. Palto, A. Sinha, A. Yasuda, S. Hashimoto, and W. Haase, Phys. Rev. E 66, 021701 (2002).

[34] J. Xue and N. A. Clark, Phys. Rev. Lett. 64, 307 (1990).

[35] K. Nakagawa, T. Shinominya, M. Koden, K. Tsubota, T. Kuratate, Y. Ishii, F. Funda, M. Matsuura, and K. Awane, Ferroelectrics 85, 39 (1988).

[36] M. S. Spector, S. K. Prasad, B. T. Weslowski, R. D. Kamien, J. V. Selinger, B. R. Ratna, and R. Shashidar, Phys. Rev. E 61,
3977 (2000).

[37] M. Terada, S. Yamada, K. Katagiri, S. Yoshihara, and J. Kanbe, Ferroelectrics 149, 283 (1993); Y. Hanyu, K. Nakamura, Y. Hotta, S. Yoshihara, and J. Kanbe, SID Int. Symp. Digest Tech. Papers 24, 364 (1993); discussed in Chap. 9.1, in S. T. Lagerwall, Ferroelectric and Antiferroelectric Liquid Crystals (Wiley-VCH, Weinheim, New York, 1999).

[38] R. Beccherelli and S. Elston, Displays 20, 573 (1998).

[39] K. D’Havé, Ph.D. thesis, Göteborg and Universiteit Gent, 2001.

[40] R. F. Shao, J. E Maclennan, N. A. Clark, D. J. Dyer, and D. M. Walba, Liq. Cryst. 28, 117 (2001).

[41] R.-F. Shao, P. C. Willis, and N. A. Clark, Ferroelectrics 121, 127 (1991).

[42] T. Rieker, N. A. Clark, G. S. Smith, D. S. Parmar, E. B. Sirota, and C. R. Safinya, Phys. Rev. Lett. 59, 2658 (1987).

[43] Simplified version of the directional filtering technique used in S. T. Lagerwall and B. Stebler, in Physique des Défauts/ Physics of Defects, edited by R. Balian, M. Kléman, and J.-P. Poirier, Les Houches Vol. XXXV (North-Holland Publishing Company, Amsterdam, 1980), pp. 757-776.

[44] J. Ogura, Casio Corp., presented at the Symposium for Celebrating the Second Anniversary of the Liquid Crystal Institute of the Science University of Tokyo (unpublished). 